\title{
Laser Guiding in the Randomicity of Discrete Surface Strengthening by Arc Discharge
}

\author{
Wang Zhitong, Zhou Zhongqiang, Han Yanliang, Shi Mao, and Yang Mingjiang
}

\begin{abstract}
Several discharge areas by laser-guided discharge (LGD) were compared with those by common arc discharge. The randomicity of discharge areas by common arc discharge was controlled by laser guiding on two scales: large scale (the spacing of the discharge areas) and small scale (the inside of the discharge area). The position of the discharge area overlapped completely with a laser focus; therefore, the distribution and surface shape of the discharge areas were controlled. The stochastic movement of anode spot in the discharge area was controlled by laser guiding. As such, the repetitive melting and solidifying of microstructures in the discharge area was constrained. The tempered microstructures in the discharge area were voided, the utilization efficiency of input energy was improved, and the strengthened depth of the discharge areas was increased. The regularity of cross-sectional shape of the discharge area was also improved. The hardness of microstructures in both discharge areas is greater than that of the base material. The highest level of hardness of microstructures in both discharge areas measures above $1000 \mathrm{HV}$. In summary, the hardness of microstructures in the discharge area by LGD is larger and more discrete than that by common arc discharge.
\end{abstract}

Index Terms-Common arc discharge, discrete surface strengthening, laser-guided discharge (LGD), tempered microstructures.

\section{INTRODUCTION}

A T PRESENT, laser-guided discharge (LGD) has been used for material processing. For instance, in 1983, Gilgenbach et al. used a $\mathrm{CO}_{2}$ pulsed laser in the atmosphere to bore some holes in aluminum foil [1]. In 1999, Hoshi and Yoshida used a 532-nm YAG pulsed laser at low air pressure to study the markings on the surface of a workpiece and the processing inside a workpiece [2], [3]. Both boring and marking by LGD are a type of removal process for many materials. However, to date, a process for a melting by LGD to strengthen the surface of materials has failed to attract much attention.

In previous works, the authors studied a method of discrete melting processing by LGD to strengthen the surface of materials [4], [5]. In this paper, discharge areas subjected to LGD are compared with those subjected to common arc discharge. The control of laser guiding in the randomicity of the discharge areas by common arc discharge is studied in terms of two scales: large scale (the spacing of the discharge areas) and small scale (the inside of the discharge area).

Manuscript received July 8, 2009; revised August 23, 2009 and January 14, 2010. First published March 8, 2010; current version published April 9, 2010. This work was supported by the National Natural Science Fund of China (60877064).

The authors are with the Key Laboratory of Mechanics in Advanced Manufacturing, Chinese Academy of Sciences, Beijing 100190, China (e-mail: ztwang@imech.ac.cn).

Digital Object Identifier 10.1109/TPS.2010.2041673

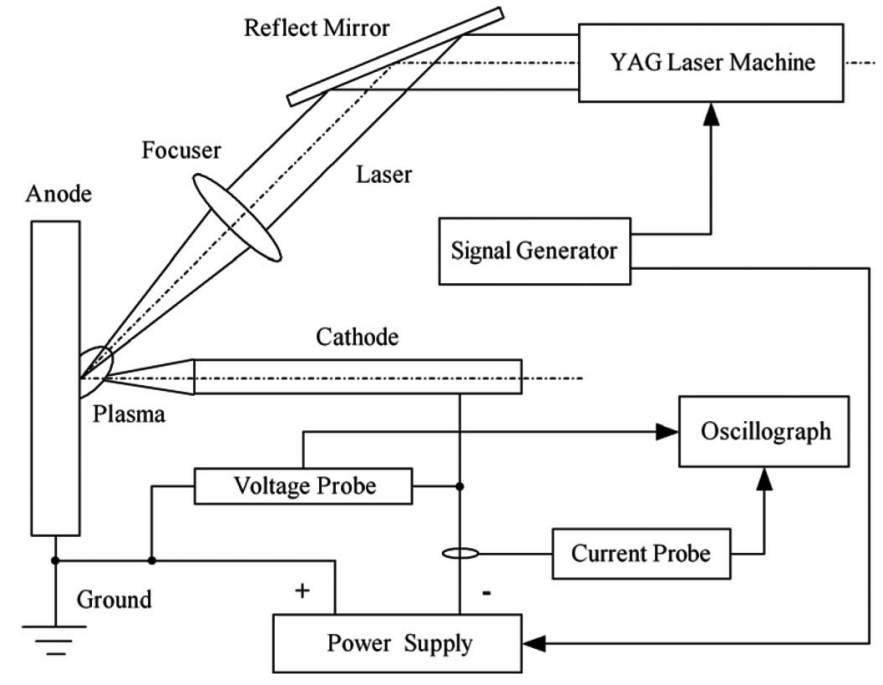

Fig. 1. Schematic of discrete surface strengthening by LGD.

\section{PRINCIPLES AND EQUiPMENT}

An experimental schematic is shown in Fig. 1. The experiment was performed in the atmosphere. A pair of electrodes in the shape of a pin and a plate was utilized. The pin was perpendicular to the plate. The plate, made of 1045 steel, was considered an anode and grounded. The pin, made of an alloy of tungsten and strontium, was used as a cathode. The diameter of the spherical tip of the pin was approximately $0.5 \mathrm{~mm}$. The surface of the plate was strengthened in the experiment. The gap between the electrodes was $0.5 \mathrm{~mm}$. Transformer oil, approximately $0.1 \mathrm{~mm}$ in thickness, was laid on the surface of the plate to restrict the expansion of the discharge area. The surface strengthened by LGD was compared with that strengthened by common arc discharge. A voltage of $4 \mathrm{kV}$ was supplied to guide the common arc discharge with a triggering probability of $100 \%$. For the LGD, a YAG laser was used with the following parameters: 10-mJ pulse energy, 120-ns pulsewidth, and $1.06-\mu \mathrm{m}$ wavelength. A laser pulse was focused on the surface of the plate to generate the metal plasma, triggering an arc discharge between the electrodes with a probability of $100 \%$. The diameter of the laser focus was approximately $160 \mu \mathrm{m}$.

The waveforms of the current and voltage were measured by the probes in the circuit and stored in the oscillograph (Tektronix TDS210). For both processes, the waveforms of the discharge current are square with a peak of approximately $100 \mathrm{~A}$ (Fig. 2). The surface photos of the discharge areas were taken by a three-dimensional (3-D) microscope (Stemi SV11). Cross-sectional samples of the discharge areas were prepared, 


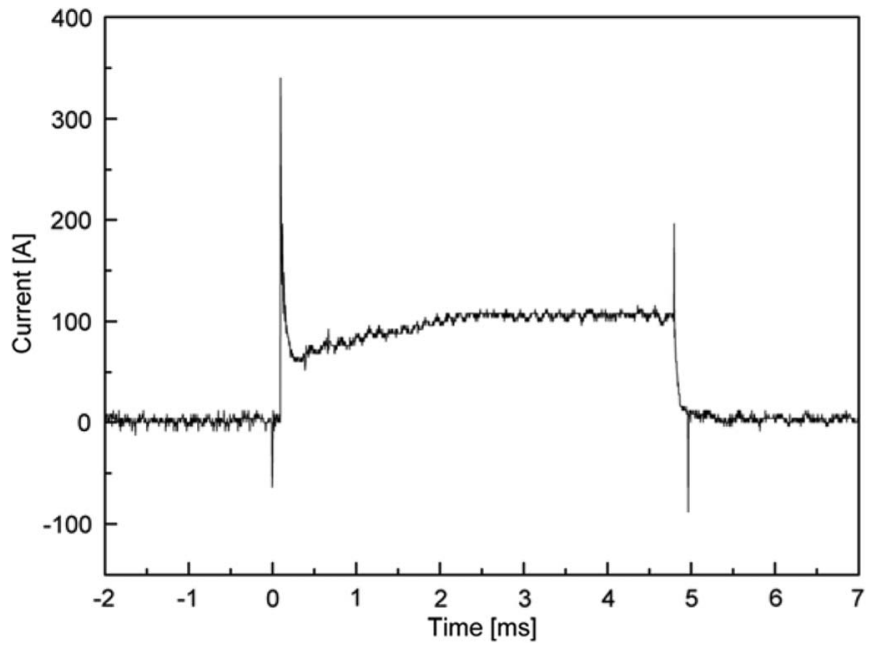

Fig. 2. Waveform of discharge current.
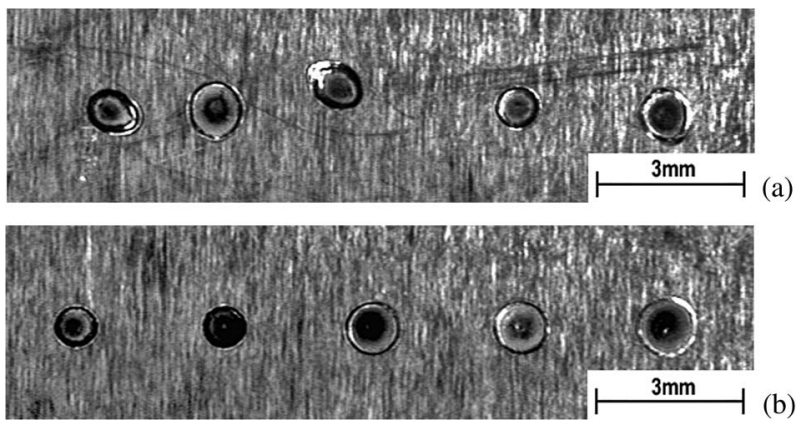

Fig. 3. Photographs of discharge areas: (a) by common arc discharge and (b) by LGD.

and optical microscope and scanning electron microscope (SEM) photographs were taken.

\section{RESULTS AND DISCUSSION}

\section{A. Laser Guiding in the Distribution and Shapes of Discrete Discharge Areas}

In the experiment, LGD and common arc discharge were separately triggered five times with a horizontal spacing of $3 \mathrm{~mm}$. The photographs of the discharge areas are shown in Fig. 3. The positions of the discharge area by common arc discharge are irregular in both the vertical and horizontal directions. The surface shapes of some discharge areas are circular, but the surface shapes of the others are elliptical. As a whole, the surface shapes of the discharge areas are irregular. Images of the discharge areas by LGD, however, are quite different. The positions of the discharge areas are regular in both directions. The surface shapes of the discharge areas are all regularly circular. Control by laser guiding in the discharge areas is shown in Fig. 4. In the process of laser guidance, the position of the discharge areas overlaps with the laser focus; therefore, the distribution of discharge areas is regular. The arc direction is perpendicular to the surface of plate. The arc pressure in the discharge area is symmetrical; therefore, the surface shapes of discharge areas are circular and regular. In contrast, in the process of common arc discharge, the position of the discharge
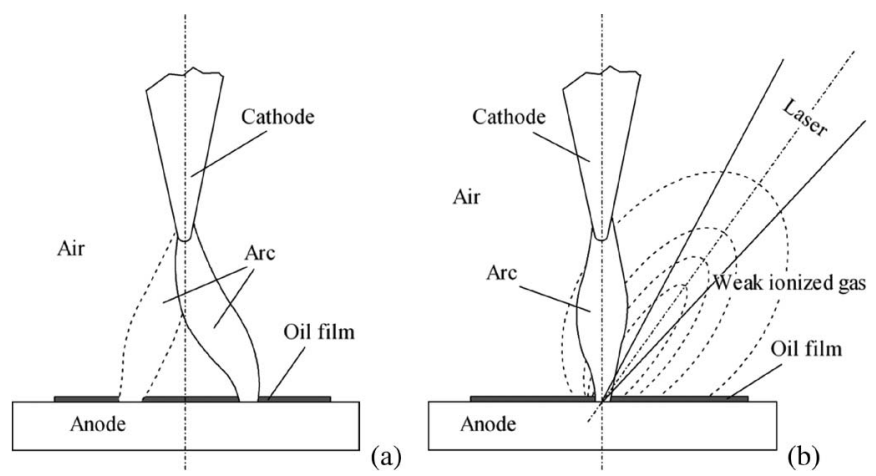

Fig. 4. Control of laser guiding in the position of the discharge area: (a) by common arc discharge and (b) by LGD.

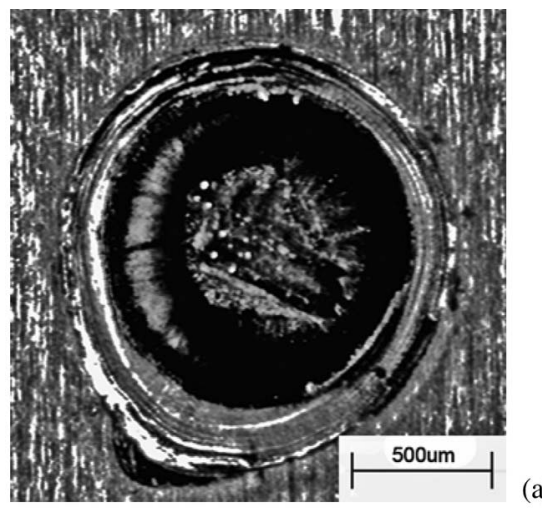

(a)

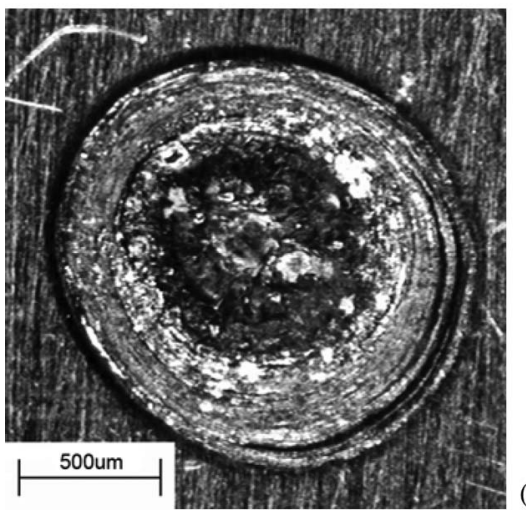

(b)

Fig. 5. Three-dimensional microscope surface photographs of discharge areas: (a) by common arc discharge and (b) by LGD.

area is stochastic decided by the surface morphology of the electrodes. When the arc direction is tilted toward the plate, the arc pressure blows the melted metal out to form an elliptical discharge area.

\section{B. Laser Guiding in the Microstructures of the Discharge Area}

In this section, both discharge areas subjected to similar experimental parameters are compared. The 3-D microscope surface photographs of the discharge areas are shown in Fig. 5. The optical microscope cross-sectional photographs of the discharge areas are also shown in Fig. 6. The experimental parameters for common arc discharge are 7.39-ms pulsewidth and 10.84-J pulse energy. Those for LGD are $7.55-\mathrm{ms}$ pulsewidth and 11-J pulse energy. The anode spot is known to exist as the stochastic movement in the process of the common arc 

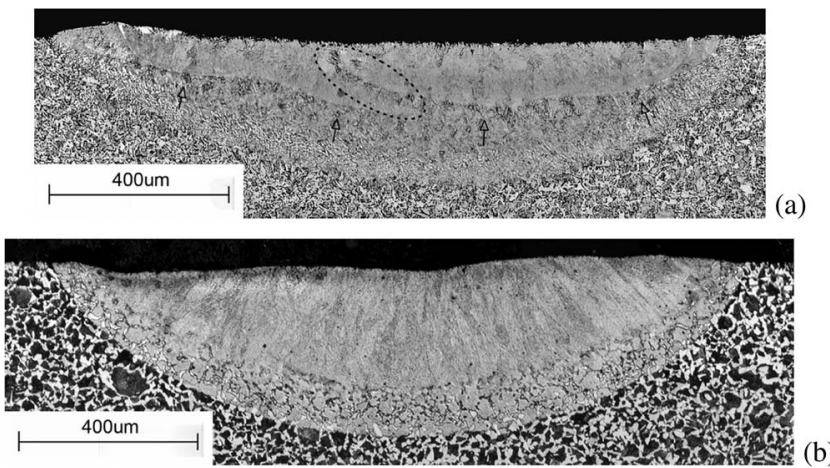

Fig. 6. Optical microscope cross-sectional photographs of discharge areas: (a) by common arc discharge and (b) by LGD.
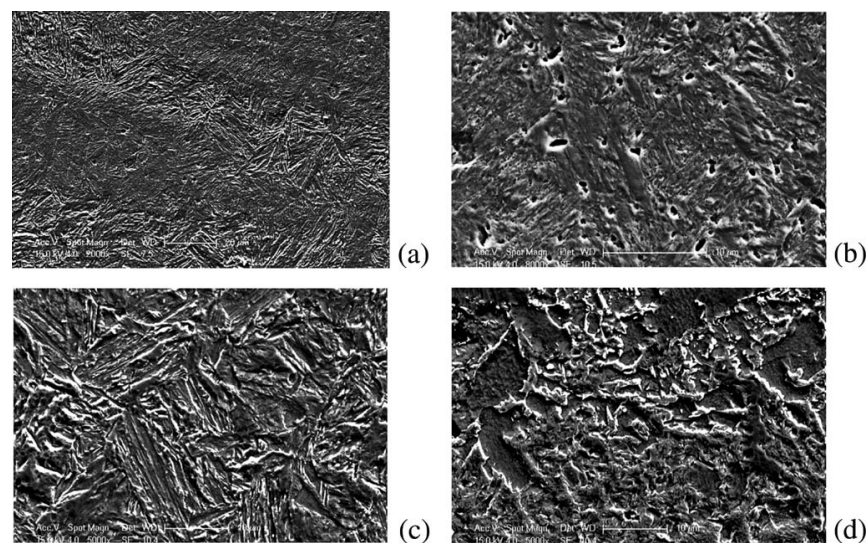

Fig. 7. SEM photographs of the microstructures in the discharge area by common arc discharge: (a) the region marked by the elliptical dashed line in Fig. 8(a); (b) $60-\mu \mathrm{m}$ below the surface; (c) $200-\mu \mathrm{m}$ below the surface; and (d) the solid-state phase transition region.

discharge [6]. The previously solidified microstructures in the discharge area are reheated by the subsequent arc, and the microstructures are remelted and tempered. When the crosssectional sample of the discharge area is prepared, the tempered microstructures [Fig. 7(c)] are eroded more severely than the solidified microstructures [Fig. 7(b)]. It is evident that the interfaces between the solidified microstructures and the tempered microstructures are formed. The interfaces are marked by the black arrowhead in Fig. 6(a). The region marked by the elliptical dashed line in Fig. 6(a) is also shown in Fig. 7(a). Two types of microstructures near the interface can be clearly identified. In the cross-sectional photograph of the microstructures of the discharge area by LGD [Fig. 6(b)], however, the same interface does not appear. The tempered microstructures were not also observed at different distances below the surface, as shown in Fig. 8(a)-(c).

The hardness of both discharge areas is measured, and the results are shown in Fig. 9. The hardness of the microstructures in the discharge area by common arc discharge is in two segments according to the depth below the surface of the base material. The first segment is within the range of $100 \mu \mathrm{m}$ below the surface. The hardness of the microstructures is approximately $1100 \mathrm{HV}$. The microstructures [Fig. 7(b)] are composed of some lath martensites, which form in the tight cell grains in a disordered arrangement. The second segment
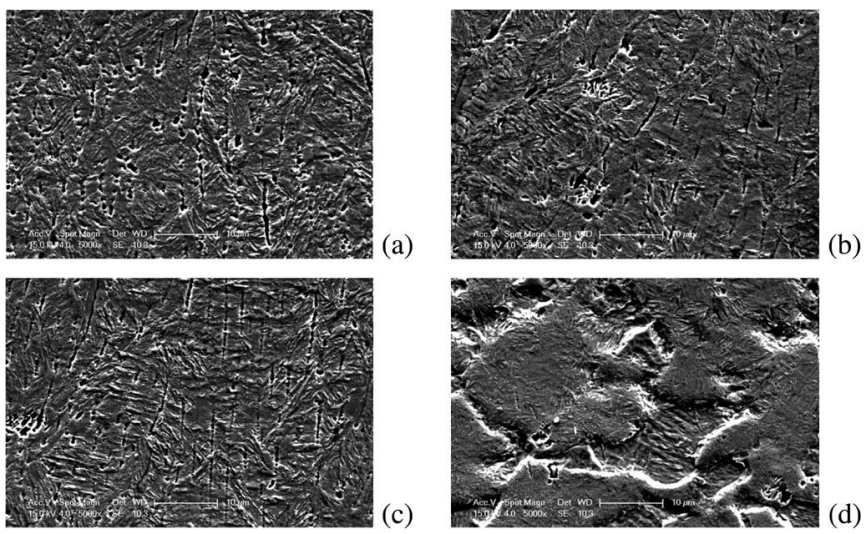

Fig. 8. SEM photographs of microstructures in the discharge area by LGD: (a) $60-\mu \mathrm{m}$ below the surface; (b) $125-\mu \mathrm{m}$ below the surface; (c) $200-\mu \mathrm{m}$ below the surface; and (d) the solid-state phase transition region.

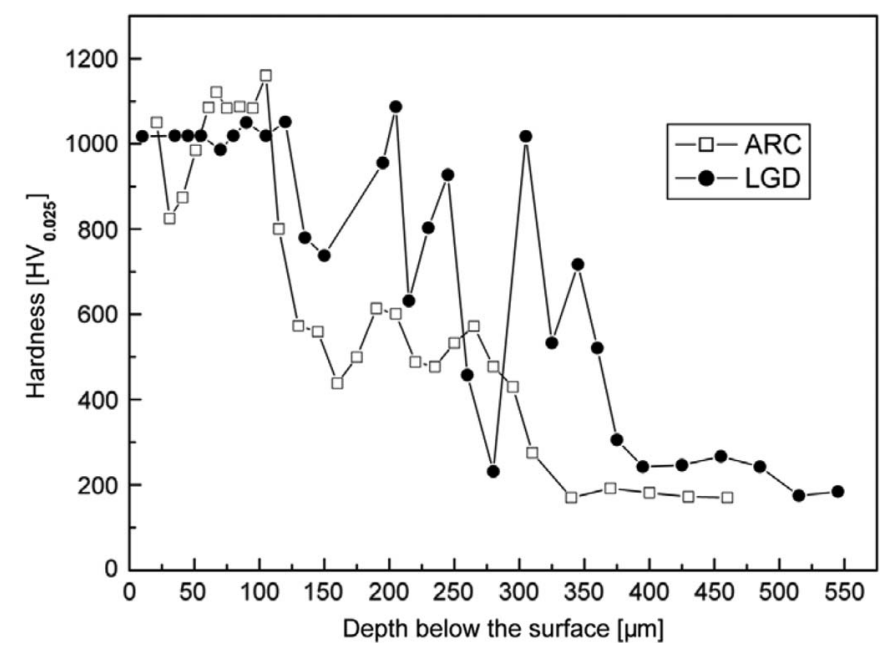

Fig. 9. Hardness of microstructures in both discharge areas.

is within the range from 100 to $300 \mu \mathrm{m}$ below the surface. The mean hardness is approximately $500 \mathrm{HV}$, with a small extent of undulation. The microstructures include some tempered martensites [Fig. 7(c)] and the microstructures of the solid-state phase transition [Fig. 7(d)].

The hardness of the microstructures in the discharge area by LGD may be divided into three segments according to the depth below the surface of the base material. The first segment is within the range of $110 \mu \mathrm{m}$ below the surface. At this depth, the hardness of the microstructures is approximately $1000 \mathrm{HV}$. The microstructures involve some lath martensites [Fig. 8(a)], which form in the dendrites in an orderly arrangement. A small quantity of retained austenites in the microstructures erodes to form the so-called valleys. The second segment is within the range from 110 to $275 \mu \mathrm{m}$ below the surface. The quantity of retained austenites in the microstructures gradually increases with the increase in depth below the surface. The quantity of valleys also increases accordingly. The extent of the hardness undulation of the microstructures in this segment is bigger than that at the same depth by common arc discharge. The third segment is within the range from 275 to $375 \mu \mathrm{m}$ in the solid-state phase transition. The microstructures here are shown in Fig. 8(d). The extent of hardness undulation of the 

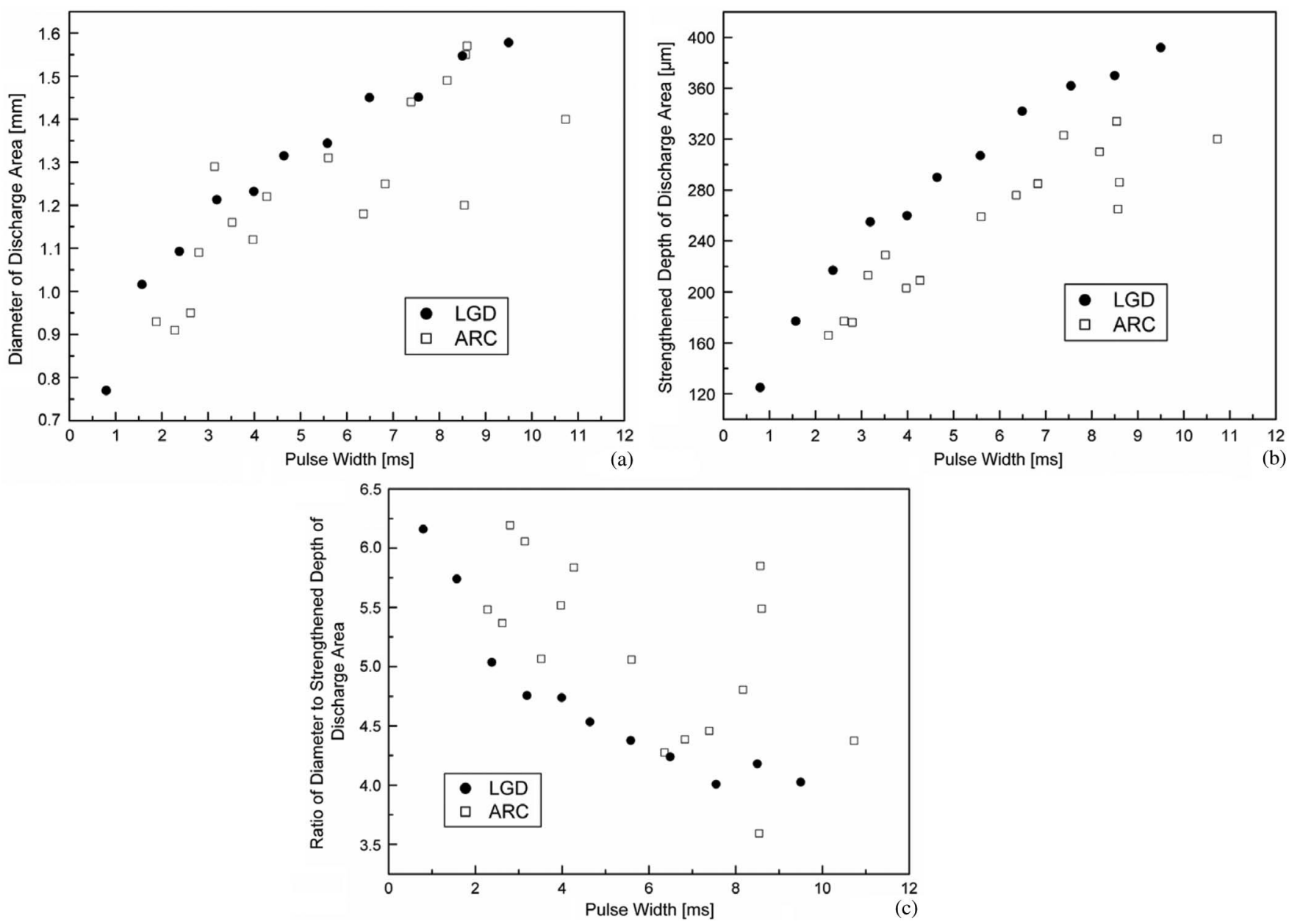

Fig. 10. Cross-sectional shape of discharge areas by LGD and that by common arc discharge: (a) diameter; (b) strengthened depth; and (c) ratio of the diameter to the strengthened depth.

microstructures in this segment is bigger than that at the second segment.

The hardness of the microstructures in both discharge areas is greater than that of the base material. In summary, the hardness of the microstructures in the discharge area by LGD is greater and more discrete than that by common arc discharge.

In the previous work, the morphology and hardness of the microstructures in the discharge area by LGD were compared with those by common arc discharge. The results highlighted the fact that laser guidance controls the stochastic movement of the anode spot in the discharge area. The repetitive melting and tempering of the microstructures in the discharge area by LGD were thus avoided.

\section{Laser Guiding in the Cross-Sectional Shapes of Discharge Areas}

In this section, the cross-sectional shapes of both discharge areas are compared. The ratio of the diameter to the strengthened depth of the discharge area is used to describe the crosssectional shape of discharge area.

The diameter of the discharge area by LGD [Fig. 10(a)] is more regular than that by common arc discharge. The strengthened depth of the discharge area by common arc discharge [Fig. 10(b)] is shallower and more discrete than that by LGD.
The ratio of the diameter to the strengthened depth of the discharge area by common arc discharge [Fig. 10(c)] is also bigger and more discrete than that by LGD.

Laser guidance controls the stochastic movement of the anode spot in the discharge area, thus improving the utilization efficiency of the input energy. As such, the strengthened depth of the discharge area is increased. For the process by common arc discharge the position of repetitive melting and tempering of microstructures in the discharge area is stochastic; therefore, the cross-sectional shapes of the discharge areas are irregular. The regularity of the cross-sectional shapes of the discharge areas by common arc discharge is improved by laser guiding.

\section{CONCLUSION}

The following conclusions were obtained from this study.

1) Laser guidance controls the position of the discharge area to overlap with the laser focus in the design. The surface shape of the discharge area is regularly circular.

2) Laser guidance constrains the stochastic movement of the anode spot in the discharge area. The repetitive melting and solidification of microstructures in the discharge area are avoided. In the discharge area by LGD, the tempered microstructures were not found. The microstructures in the discharge area may be divided into two regions: the 
melted region and the solid-state phase transition region. The microstructures in the melted region are combined with some lath mantensites formed in the regular dendrites and a small quantity of retained austenites.

3) The hardness of the microstructures in both discharge areas is greater than that of the base material. The highest level of hardness of the microstructures in both discharge areas measures over $1000 \mathrm{HV}$. In summary, the hardness of the microstructures in the discharge area by LGD is greater and more discrete than that by common arc discharge.

4) The wastage of the input energy is prevented by laser guiding, thereby increasing the strengthened depth of the discharge area and improving the regularity of the crosssectional shape of the discharge area.

\section{REFERENCES}

[1] R. M. Gilgenbach, O. E. Ulrich, and L. D. Horton, "Localized metallic melting and hole boring by laser guided discharges," Rev. Sci. Instrum., vol. 54, no. 1, pp. 109-113, Jan. 1983.

[2] Y. Hoshi and H. Yoshida, "Application of laser-guided discharge to processing," Appl. Phys. A, Solids Surf., vol. 68, no. 1, pp. 93-98, Jan. 1999.

[3] Y. Hoshi, H. Yoshida, and Y. Tsutsui, "Electric discharge image marking using laser guided discharge," IEEE Trans. Plasma Sci., vol. 28, no. 5, pp. 1771-1774, Oct. 2000 .

[4] W. Zhitong, Z. Jian, and Y. Mingjiang, "Effect of surface condition of electrode on the surface melting of material by laser-guided micro-arc discharge," IEEE Trans. Plasma Sci., vol. 36, no. 5, pp. 2816-2819, Oct. 2008.

[5] W. Zhitong, Y. Mingjiang, S. Mao, and H. Yanliang, "Surface strengthening of 45\# steel by laser-guided micro discharge," Chin. J. Lasers, vol. 36, pp. 2178-2181, Aug. 2009.

[6] J. Huangzhong, Ed., Arc Welding \& Electro-Slag Welding. China: Chin. Mach. Press, Nov. 1988.

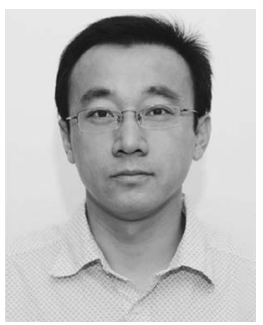

Wang Zhitong was born in China, in 1972. He received the Ph.D. degree from the Institute of Mechanics, Chinese Academy of Sciences, Beijing, China.

He is currently affiliated with the National Center for Laser-Texturing Technology Development and Application, Key Laboratory for Mechanics in Advanced Manufacturing, the Institute of Mechanics, Chinese Academy of Sciences. His main research work is about material surface modification. He has ing by laser-guided discharge in the atmosphere.

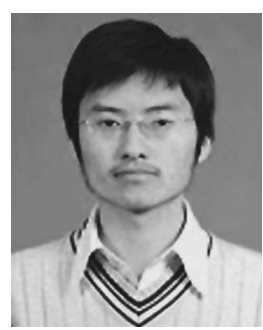

Zhou Zhongqiang was born in China, in 1985 . He received the M.S. degree from the Institute of the Mechanics, Chinese Academy of Sciences, Beijing, China.

His major research interest is about the FEM simulation of material surface strengthening by laserguided discharge in atmosphere.

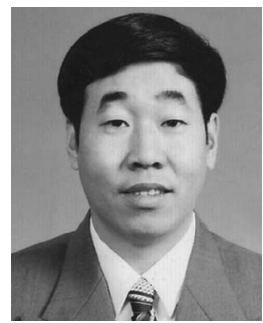

Han Yanliang was born in China, in 1954. He received the B.S. degree from the University of Science and Technology of China, Hefei, China.

$\mathrm{He}$ is currently affiliated with the National Center for Laser-Texturing Technology Development and Application, Key Laboratory for Mechanics in Advanced Manufacturing, the Institute of Mechanics, Chinese Academy of Sciences, Beijing, China.

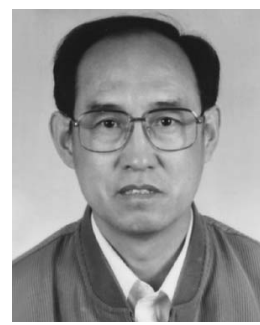

Shi Mao was born in China, in 1942. He received the Associate degree from the Chinese Academy of Sciences, Beijing, China.

$\mathrm{He}$ is currently affiliated with the National Center for Laser-Texturing Technology Development and Application, Key Laboratory for Mechanics in Advanced Manufacturing, the Institute of Mechanics, Chinese Academy of Sciences, Beijing, China.

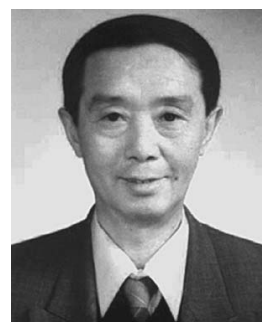

Yang Mingjiang was born in China, in 1944. He received the Ph.D. degree from the Institute of Mechanics, Chinese Academy of Sciences, Beijing, China.

He is currently affiliated with the National Center for Laser-Texturing Technology Development and Application, Key Laboratory for Mechanics in Advanced Manufacturing, the Institute of Mechanics, Chinese Academy of Sciences. He is the Chief of the National Center for Laser-Texturing Technology Development and Application. He improved the technique of YAG laser-texturing, and applied this technique to the cold-roll industry. 